\title{
The Effects Of Intra-Articular Platelet-Rich Plasma Injections Versus Corticosteroid Injections On Pain Score And Function In Knee Osteoarthritis
}

\author{
N. Bagatir ${ }^{1}$, C. Cinar ${ }^{1}$, Y. Soluk Ozdemir ${ }^{1}$, A.N. Bardak ${ }^{1}$, K. Ones ${ }^{1}$, A.Metin Terzibasioglu ${ }^{1}$ \\ ${ }^{1}$ University of Health Sciences Istanbul Physical Therapy And Rehabilitation Training And Research Hospital
}

Background and aims: Osteoarthritis is a chronic joint disease that leads to pain and disabilities (1). The aim of this preliminary study is examine the effects of platelet-rich plasma (PRP) injection and corticosteroid (CS) injections on the level of pain and function of the knee joint in patients with $O A$.

Methods: The participants who suffered from knee osteoarthritis were randomly divided into two groups: intra articular injection of PRP and CS. Western Ontario and McMaster Universities Osteoarthritis Index (WOMAC), Short Form-36 (SF-36), ranges of motions (ROM) of knee and pain intensity based on Visual Analog Scale (VAS) rest and motion were assessed before the treatment and four weeks after the treatment. Also knee cartilage thickness were measured by ultrasonography in both groups.

Results: Forty participants were involved in this study that group 1 (corticosteroid injections) was included 19 patients and group 2 (PRP injections) was 21 patients. VAS rest - VAS motion and WOMAC scores were significantly decreased and ROM flexion, SF-36 scores and knee cartilage thickness measurement were significantly increased in both groups at first month of clinical outcomes, but there were no significantly difference between the two groups.

Conclusion: Both PRP and CS injections appears to be effective in symptomatic knee osteoarthritis. We found that there were no superiority of PRP treatment to CS treatment at short time follow up measures, but PRP injections may be show superior clinical outcomes to reduce pain and improvement in the quality of life of patients in the long term when compared with CS injections.

\section{References}

1. Neogi T. The Epidemiology and Impact of Pain in Osteoarthritis. Osteoarthritis Cartilage. 2013 Sep;21(9):1145-53.
Table 1

\begin{tabular}{|c|c|c|c|c|c|c|c|c|}
\hline \multirow[b]{2}{*}{ Group 1} & & \multicolumn{3}{|c|}{ Pre-treatment } & \multicolumn{3}{|c|}{ Post-treatment } & \multirow[b]{2}{*}{$\mathrm{p}$} \\
\hline & & $M \pm$ S.D. $/ n-\%$ & & $\mathrm{~d}($ Min-Max $)$ & $M \pm$ S.D. $/ \mathrm{n}-\%$ & & $($ Min-Max) & \\
\hline VAS-rest & & $3,5 \pm 2,6$ & 5 & $(0-8)$ & $1,4 \pm 2,1$ & 0 & $(0-6)$ & $0,005^{*}$ \\
\hline VAS-motion & & $6,3 \pm 1,8$ & 7 & $(2-8)$ & $3,2 \pm 2,6$ & 2 & $(0-8)$ & $0,001^{*}$ \\
\hline \multirow{2}{*}{ ROM-extension } & Limited & 3 & & $16 \%$ & 1 & & $5 \%$ & \multirow{2}{*}{0,500} \\
\hline & Not limited & 16 & & $84 \%$ & 18 & & $95 \%$ & \\
\hline \multirow{2}{*}{ ROM-flexion } & Limited & 12 & & $63 \%$ & 5 & & $26 \%$ & \multirow{2}{*}{$0,016^{*}$} \\
\hline & Not limited & 7 & & $37 \%$ & 14 & & $74 \%$ & \\
\hline \multicolumn{9}{|l|}{ Group 2} \\
\hline VAS-rest & & $3,3 \pm 2,7$ & 4 & $(0-8)$ & $1,1 \pm 1,8$ & 0 & $(0-8)$ & $0,001^{*}$ \\
\hline VAS-motion & & $8,0 \pm 1,1$ & 8 & $(5-10)$ & $3,9 \pm 2,1$ & 4 & $(0-8)$ & $0,000^{*}$ \\
\hline \multirow{2}{*}{ ROM-extension } & Limited & 1 & & $5 \%$ & 1 & & $5 \%$ & \multirow{2}{*}{1,000} \\
\hline & Not limited & 20 & & $95 \%$ & 20 & & $95 \%$ & \\
\hline \multirow[b]{2}{*}{ ROM-flexion } & Limited & 13 & & $62 \%$ & 4 & & $19 \%$ & \multirow{2}{*}{$0,004^{*}$} \\
\hline & Not limited & 8 & & $38 \%$ & 17 & & $81 \%$ & \\
\hline
\end{tabular}

Table 2

\begin{tabular}{|c|c|c|c|c|c|c|}
\hline \multirow{3}{*}{ Pre-treatment } & & \multicolumn{3}{|c|}{ Group 1} & Group 2 & \multirow[b]{2}{*}{$\mathrm{p}$} \\
\hline & & \multicolumn{2}{|c|}{$M \pm S . D . / n-\%$} & \multirow[t]{2}{*}{ Med(Min- } & $\mathrm{M} \pm$ S.D./n-\% $\operatorname{Med}(\operatorname{Min}-\mathrm{Max})$ & \\
\hline & & & & & & \\
\hline VAS-rest & & 3,5 & $\pm 2,6$ & $5 \quad(0-8)$ & $3,3 \pm 2,7 \quad 4 \quad(0-8)$ & 0,859 \\
\hline VAS-motion & & 6,3 & $\pm \quad 1,8$ & $\begin{array}{ll}7 \quad(2-8) \\
\end{array}$ & $1,1 \pm 1,8 \quad 0 \quad(0-8)$ & 0,552 \\
\hline ROM-extension & Limited & 3 & $16 \%$ & & $\begin{array}{ll}1 & 5 \% \\
\end{array}$ & 0,331 \\
\hline & Not limited & 16 & $84 \%$ & & $95 \%$ & \\
\hline ROM-flexion & $\begin{array}{r}\text { Limited } \\
\text { Not limited }\end{array}$ & $\begin{array}{c}12 \\
7\end{array}$ & $\begin{array}{l}63 \% \\
37 \%\end{array}$ & & $\begin{array}{cc}13 & 62 \% \\
8 & 38 \%\end{array}$ & 0,935 \\
\hline Post-treatment & & & & & & \\
\hline VAS-rest & & 1,4 & 2,1 & $0 \quad(0-6)$ & $8,0 \pm 1,18$ & 0,050 \\
\hline VAS-motion & & $\begin{array}{l}1,7 \\
3,2\end{array}$ & $\pm \quad 2,6$ & $2 \quad(0-8)$ & $3,9 \pm 2,1 \quad 4 \quad(0-8)$ & 0,211 \\
\hline ROM-extension & Limited & 1 & $5 \%$ & & $\begin{array}{ll}1 & 5 \%\end{array}$ & 1,000 \\
\hline & Not limited & 18 & $95 \%$ & & $95 \%$ & \\
\hline & Limited & 5 & $26 \%$ & & $19 \%$ & \\
\hline ROM-flexion & Not limited & 14 & $74 \%$ & & $81 \%$ & 0,712 \\
\hline
\end{tabular}

Table 3

\begin{tabular}{|c|c|c|c|c|c|c|}
\hline \multirow[b]{3}{*}{ Pre-treatment SF-36 } & \multicolumn{3}{|l|}{ Group 1} & \multicolumn{2}{|l|}{ Group 2} & \multirow[b]{2}{*}{ p } \\
\hline & $M \pm$ S.D. $/ \mathrm{n}-\%$ & \multicolumn{2}{|c|}{$\operatorname{Med}(\operatorname{Min}-\operatorname{Max})$} & \multirow[t]{2}{*}{$M \pm$ S.D. $/ \mathrm{n}-\%$} & $\operatorname{Med}(\operatorname{Min}-\operatorname{Max})$ & \\
\hline & & & & & & \\
\hline Physical functioning & $44,4 \pm 24,7$ & 40 & $(5-95)$ & $45,0 \pm 20,8$ & $(10-85)$ & 0,942 \\
\hline Physical Role limitations & $32,8 \pm 32,3$ & 25 & $(0-100)$ & $38,0 \pm 38,4$ & $25 \quad(0-100)$ & 0,811 \\
\hline Emotional Role limitations & $29,7 \pm 39,8$ & 0 & $(0-100)$ & $36,4 \pm 40,7$ & $33(0-100)$ & 0,550 \\
\hline Energy/fatigue & $35,5 \pm 21,2$ & 30 & $(10-90)$ & $39,7 \pm 21,2$ & $40 \quad(0-85)$ & 0,532 \\
\hline Emotional well-being & $44,2 \pm 17,0$ & 44 & $(16-72)$ & $50,6 \pm 18,4$ & $52 \quad(16-80)$ & 0,259 \\
\hline Social functioning & $54,1 \pm 24,2$ & 50 & $(25-100)$ & $58,0 \pm 24,7$ & $63 \quad(13-100)$ & 0,611 \\
\hline Pain & $33,0 \pm 26,1$ & 23 & $(0-90)$ & $28,3 \pm 19,4$ & $23 \quad(0-78)$ & 0,691 \\
\hline General health & $46,5 \pm 22,7$ & 45 & $(0-95)$ & $37,8 \pm 17,8$ & $35 \quad(0-70)$ & 0,184 \\
\hline $\begin{array}{l}\text { Pre-treatment USG } \\
\text { measurement }\end{array}$ & $1,99 \pm 0,39$ & 2,00 & $(1,43-2,96)$ & $1,96 \pm 0,44$ & $1,93(1,06-2,76)$ & 0,849 \\
\hline Pre-treatment WOMAC & $50,6 \pm 19,7$ & 54,1 & $(3,1-77)$ & $59,0 \pm 22,0$ & $58,3(30,2-129)$ & 0,212 \\
\hline \multicolumn{7}{|l|}{ Post-treatment SF-36 } \\
\hline Physical functioning & $66,0 \pm 23,8$ & 70 & $(15-100)$ & $53,5 \pm 24,1$ & $45 \quad(5-100)$ & 0,109 \\
\hline Physical Role limitations & $64,4 \pm 32,6$ & 75 & $(0-100)$ & $54,7 \pm 42,2$ & $50 \quad(0-100)$ & 0,529 \\
\hline Emotional Role limitations & $59,6 \pm 45,2$ & 67 & $(0-100)$ & $55,5 \pm 48,7$ & $100(0-100)$ & 0,929 \\
\hline Energy/fatigue & $46,0 \pm 25,8$ & 40 & $(5-100)$ & $41,6 \pm 20,1$ & $40 \quad(0-100)$ & 0,551 \\
\hline Emotional well-being & $55,7 \pm 17,3$ & 56 & $(20-84)$ & $49,1 \pm 18,3$ & $48 \quad(0-76)$ & 0,248 \\
\hline Social functioning & $73,2 \pm 19,9$ & 75 & $(38-100)$ & $63,8 \pm 25,5$ & $63 \quad(25-100)$ & 0,206 \\
\hline Pain & $59,3 \pm 25,3$ & 68 & $(10-100)$ & $52,0 \pm 21,0$ & $45 \quad(10-90)$ & 0,325 \\
\hline General health & $52,1 \pm 25,2$ & 55 & $(5-100)$ & $40,4 \pm 14,9$ & $(0-65)$ & 0,091 \\
\hline $\begin{array}{l}\text { Post-treatment USG } \\
\text { measurement }\end{array}$ & $2,23 \pm 0,56$ & 2,26 & $(0,96-3,16)$ & $2,26 \pm 0,33$ & $2,23(1,80-2,96)$ & 0,870 \\
\hline Post-treatment WOMAC & $34,4 \pm 23,5$ & 35,4 & $(0-77)$ & $33,6 \pm 17,8$ & $29,1(1,7-84)$ & 0,912 \\
\hline
\end{tabular}

Table 4

\begin{tabular}{|c|c|c|c|c|c|c|c|}
\hline Group 1 & $\frac{\text { Pre-treatment }}{M \pm S, D}$ & $\frac{\text { Post-treatment }}{M \pm S . D}$ & $p$ & Group 2 & $\frac{\text { Pre-treatment }}{M \pm S . D .}$ & $\frac{\text { Post-treatment }}{M \pm S . D .}$ & $p$ \\
\hline SF-36 & & & & SF-36 & & & \\
\hline Physical functioning & $44,4 \pm 24,7$ & $66,0 \pm 23,8$ & $0,001^{*}$ & Physical functioning & $45,0 \pm 20,8$ & $53,5 \pm 24,1$ & 0,086 \\
\hline Physical Role limitations & $32,8 \pm 32,3$ & $64,4 \pm 32,6$ & $0,017^{*}$ & Physical Role limitations & $38,0 \pm 38,4$ & $54,7 \pm 42,2$ & 0,140 \\
\hline Emotional Role limitations & $29,7 \pm 39,8$ & $59,6 \pm 45,2$ & $0,010^{*}$ & Emotional Role limitations & $36,4 \pm 40,7$ & $55,5 \pm 48,7$ & 0,102 \\
\hline Energy/fatigue & $35,5 \pm 21,2$ & $46,0 \pm 25,8$ & $0,072^{*}$ & Energy/fatigue & $39,7 \pm 21,2$ & $41,6 \pm 20,1$ & 0,635 \\
\hline Emotional well-being & $44,2 \pm 17,0$ & $55,7 \pm 17,3$ & $0,032^{*}$ & Emotional well-being & $50,6 \pm 18,4$ & $49,1 \pm 18,3$ & 0,616 \\
\hline Social functioning & $54,1 \pm 24,2$ & $73,2 \pm 19,9$ & $0,001^{*}$ & Social functioning & $58,0 \pm 24,7$ & $63,8 \pm 25,5$ & 0,365 \\
\hline Pain & $33,0 \pm 26,1$ & $59,3 \pm 25,3$ & $0,000^{*}$ & Pain & $28,3 \pm 19,4$ & $52,0 \pm 21,0$ & $0,000^{*}$ \\
\hline General health & $46,5 \pm 22,7$ & $52,1 \pm 25,2$ & 0,171 & General health & $37,8 \pm 17,8$ & $40,4 \pm 14,9$ & 0,205 \\
\hline USG measurement & $1,99 \pm 0,39$ & $2,23 \pm 0,56$ & $0,017^{*}$ & USG measurement & $1,96 \pm 0,44$ & $2,26 \pm 0,33$ & $0,000^{*}$ \\
\hline WOMAC & $50,6 \pm 19,7$ & $34,4 \pm 23,5$ & $0,005^{*}$ & WOMAC & $59,0 \pm 22,0$ & $33,6 \pm 17,8$ & $0,000^{*}$ \\
\hline
\end{tabular}

